\title{
Empirical Likelihood for Partial Parameters in ARMA Models with Infinite Variance
}

\author{
Jinyu Li, ${ }^{1}$ Wei Liang, ${ }^{2}$ and Shuyuan $\mathrm{He}^{3}$ \\ ${ }^{1}$ School of Sciences, China University of Mining and Technology, Xuzhou 221116, China \\ ${ }^{2}$ School of Mathematical Sciences, Xiamen University, Xiamen 361005, China \\ ${ }^{3}$ School of Mathematical Sciences, Capital Normal University, Beijing 100048, China \\ Correspondence should be addressed to Jinyu Li; ljycumt@163.com
}

Received 31 March 2014; Accepted 24 August 2014; Published 2 September 2014

Academic Editor: Charalampos Tsitouras

Copyright (c) 2014 Jinyu Li et al. This is an open access article distributed under the Creative Commons Attribution License, which permits unrestricted use, distribution, and reproduction in any medium, provided the original work is properly cited.

This paper proposes a profile empirical likelihood for the partial parameters in $\operatorname{ARMA}(p, q)$ models with infinite variance. We introduce a smoothed empirical log-likelihood ratio statistic. Also, the paper proves a nonparametric version of Wilks's theorem. Furthermore, we conduct a simulation to illustrate the performance of the proposed method.

\section{Introduction}

Consider the stationary $\operatorname{ARMA}(p, q)$ time series $\left\{y_{t}\right\}$ generated by

$$
\begin{aligned}
y_{t}= & \varphi_{1} y_{t-1}+\cdots+\varphi_{p} y_{t-p} \\
& +\varepsilon_{t}+\vartheta_{1} \varepsilon_{t-1}+\cdots+\vartheta_{q} \varepsilon_{t-q},
\end{aligned}
$$

where the innovation process $\left\{\varepsilon_{t}\right\}$ is a sequence of i.i.d. random variables. When $E\left(\varepsilon_{t}^{2}\right)=\infty$, model (1) is an infinite variance autoregressive moving average (IVARMA) model, which defines a heavy-tailed process $\left\{y_{t}\right\}$. For model (1), statistical inference has been explored in many studies (see, e.g., $[1,2])$. Recently, for example, Pan et al. [3] and Zhu and Ling [4] proposed a weighted least absolute deviations estimator (WLADE) for model (1) and obtained the asymptotic normality.

However, in the building of ARMA models, we are usually only interested in statistical inference for partial parameters. For example, in the sparse coefficient (a part of zero coefficients) ARMA models, it is necessary to determine which coefficient is zero. For model (1), one traditional method is to construct confidence regions for the partial parameters of interest by normal approximation as in [3]. However, since the limit distribution depends on the unknown nuisance parameters and density function of the errors, estimating the asymptotic variance is not a trivial task. Based on these, this paper tries to put forward a new method for the estimation of partial parameters of ARMA models. We propose an empirical likelihood method, which was introduced by Owen $[5,6]$. Based on the estimating equations of WLADE, a smoothed profile empirical likelihood ratio statistic is derived, and a nonparametric version of Wilks's theorem is proved. Therefore, we can construct confidence regions for the partial parameters of interest. Also, simulations suggest that, for relative small sample cases, the empirical likelihood confidence regions are more accurate than those confidence regions constructed by the normal approximation based on the WLADE proposed by Pan et al. [3].

As an effective nonparametric inference method, the empirical likelihood method produces confidence regions whose shape and orientation are determined entirely by the data and therefore avoids secondary estimation. In the past two decades, the empirical likelihood method has been extended to many applications [7]. There are also many studies of empirical likelihood method for autoregressive models. Monti [8] considered the empirical likelihood in the frequency domain; Chuang and Chan [9] developed the empirical likelihood for unstable autoregressive models with innovations being a martingale difference sequence with finite variance; Chan et al. [10] applied the empirical 
likelihood to near unit root $\mathrm{AR}(1)$ model with infinite variance errors; Li et al. [11, 12], respectively, used the empirical likelihood to infinite variance $\operatorname{AR}(p)$ models and model (1).

The rest of the paper is organized as follows. In Section 2, we propose the profile empirical likelihood for the parameters of interest and show the main result. Section 3 provides the proofs of the main results. Some simulations are conducted in Section 4 to illustrate our approach. Conclusions are given in Section 5.

\section{Methodology and Main Results}

First, the parameter space is denoted by $\Theta \subset R^{p+q}$, which contains the true value $\theta_{0}$ of the parameter $\theta$ as an inner point. For $\theta=\left(\varphi_{1}, \ldots, \varphi_{p}, \vartheta_{1}, \ldots, \vartheta_{q}\right)$, put

$$
\varepsilon_{t}(\theta)= \begin{cases}y_{t}-\sum_{i=1}^{p} \varphi_{i} y_{t-i}-\sum_{j=1}^{q} \vartheta_{j} \varepsilon_{t-j}(\theta), & \text { if } t>0, \\ 0, & \text { if } t \leq 0,\end{cases}
$$

where $y_{t} \equiv 0$ for all $t \leq 0$, and note that $\varepsilon_{t}\left(\theta_{0}\right) \neq \varepsilon_{t}$, because of this truncation.

We define the objective function as

$$
S_{n}(\theta)=\sum_{t=u+1}^{n} \widetilde{w}_{t}\left|\varepsilon_{t}(\theta)\right|,
$$

where $u \geq \max (p, q)$ and the weight function $\widetilde{w}_{t}=1 /(1+$ $\left.\sum_{k=1}^{t-1} k^{-\alpha}\left|y_{t-k}\right|\right)^{4}$, depending on a constant $\alpha>2$. The WLADE, denoted by $\hat{\theta}$, is a lacol minimizer of $S_{n}(\theta)$ in a neighborhood of $\theta_{0}$ [3]. Denote $A_{t}(\theta)=\left(A_{t, 1}(\theta), \ldots\right.$, $\left.A_{t, p+q}(\theta)\right)^{\tau}$, where $A_{t, i}(\theta)=-\partial \varepsilon_{t}(\theta) / \partial \theta_{i}$. By (8.11.9) of Brockwell and Davis [13], it holds for $t>\max (p, q)$ that

$$
\begin{gathered}
A_{t, i}(\theta)+\sum_{j=1}^{q} \vartheta_{j} A_{t-j, i}(\theta)=y_{t-i}, \quad i=1, \ldots, p, \\
A_{t, i+p}(\theta)+\sum_{j=1}^{q} \vartheta_{j} A_{t-j, i+p}(\theta)=\varepsilon_{t-i}(\theta), \quad i=1, \ldots, q .
\end{gathered}
$$

Hence, $\widehat{\theta}$ satisfies estimating equation

$$
\frac{1}{n-u} \sum_{t=u+1}^{n} \widetilde{w}_{t} A_{t}(\theta) \operatorname{sgn}\left\{\varepsilon_{t}(\theta)\right\}=0,
$$

where $\operatorname{sgn}(x)=-1$ for $x<0$ and $=1$ for $x \geq 0$ (see [14]). Note that the above estimating equation is not differentiable at point $\theta$ such that $\varepsilon_{t}(\theta)=0$ for some $t$. This causes some problems for our subsequent asymptotic analysis. To overcome this problem, we replace it with a smooth function. Define a probability density kernel $K(\cdot)$ [15] such that $\int_{-\infty}^{+\infty} x^{j} K(x) d x=0, \kappa$ for $j=1,2$, respectively, where $\kappa \neq 0$. Let $G_{h}(x)=\int_{-x / h}^{x / h} K(u) d u$ for $h>0$. Then, a smoothed version of (5) is

$$
\frac{1}{n-u} \sum_{t=u+1}^{n} \widetilde{w}_{t} A_{t}(\theta) G_{h}\left(\varepsilon_{t}(\theta)\right)=0 .
$$

Let $m_{t h}(\theta)=\widetilde{w}_{t} A_{t}(\theta) G_{h}\left(\varepsilon_{t}(\theta)\right)$; a smoothed empirical log-likelihood ratio is defined as

$l_{h}(\theta)$

$$
\begin{array}{r}
=-2 \sup \left\{\sum_{t=u+1}^{n} \log \left((n-u) p_{t}\right) \mid \sum_{t=u+1}^{n} p_{t} m_{t h}(\theta)=0,\right. \\
\left.p_{t} \geq 0, \sum_{t=u+1}^{n} p_{t}=1\right\} .
\end{array}
$$

Using the Lagrange multiplier, the optimal value of $p_{t}$ is derived to be

$$
\begin{array}{r}
p_{t}(\theta)=\frac{1}{(n-u)\left(1+\lambda(\theta)^{\tau} m_{t h}(\theta)\right)}, \\
u+1 \leq t \leq n,
\end{array}
$$

where $\lambda(\theta)$ is a $p+q$-dimensional vector of Lagrange multipliers satisfying

$$
\frac{1}{n-u} \sum_{t=u+1}^{n} \frac{m_{t h}(\theta)}{1+\lambda(\theta)^{\tau} m_{t h}(\theta)}=0 .
$$

This gives the smoothed empirical log-likelihood ratio statistic

$$
l_{h}(\theta)=2 \sum_{t=u+1}^{n} \log \left(1+\lambda(\theta)^{\tau} m_{t h}(\theta)\right) .
$$

Let $\theta=\left(\phi^{\tau}, \omega^{\tau}\right)^{\tau}$, where $\omega \in R^{m}(1 \leq m \leq p+q)$ is the parameter of interest and $\phi \in R^{p+q-m}$ is the nuisance parameter. Note that $m=p+q$ means no nuisance parameters. Let $\phi_{0}$ and $\omega_{0}$ denote the true values of $\phi$ and $\omega$, respectively. The profile empirical likelihood is defined as

$$
l_{p}(\omega)=\min _{\phi} l_{h}(\phi, \omega)
$$

That is, $l_{p}(\omega)=l_{h}(\widetilde{\phi}(\omega), \omega)$, where $\tilde{\phi}=\tilde{\phi}(\omega):=\arg \min _{\phi} l_{h}$ $(\phi, \omega)$.

The following conditions are in order.

(A1) The characteristic polynomial $\phi(z)=1-\varphi_{1} z-\cdots-$ $\varphi_{p} z^{p}$ and $\theta(z)=1+\vartheta_{1} z+\cdots+\vartheta_{q} z^{q}$ have no common zeros, and all roots of $\phi(z)$ and $\theta(z)$ are outside the unit circle.

(A2) The innovation $\left\{\varepsilon_{t}\right\}$ has zero median and a differentiable density $f(x)$ satisfying the conditions $f(0)>0$, $\sup _{x \in R}|f(x)|<B_{1}<\infty$, and $\sup _{x \in R}\left|f^{\prime}(x)\right|<B_{2}<$ $\infty$. Furthermore, $E\left|\varepsilon_{t}\right|^{\delta}<\infty$ for some $\delta>0$, and $\alpha>\max \{2,2 / \delta\}$.

(A3) As $n \rightarrow \infty, u \rightarrow \infty$ and $u / n \rightarrow 0$.

(A4) The second derivative of $K$ exists in $R$ and $K^{\prime}(x)$ and $K^{\prime \prime}(x)$ are bounded.

(A5) $h=1 / n^{\gamma}$ with $1 / 4<\gamma<1 / 3$.

First we show the existence and consistency of $\widetilde{\phi}\left(\omega_{0}\right)$. 
Proposition 1. Let $d_{n}=1 / n^{\beta}$ with $\max \{1 / 3,3 \gamma / 2\}<\beta<$ $1 / 2$. Assume (A1)-(A5) hold; then as $n \rightarrow \infty$, with probability 1 , there exists a local minimizer $\tilde{\phi}$ of $l_{h}\left(\phi, \omega_{0}\right)$ which lies in the interior of the ball $B=\left\{\phi:\left\|\phi-\phi_{0}\right\| \leq d_{n}\right\}$. Moreover $\widetilde{\phi}$ and $\widetilde{\lambda}=\lambda\left(\widetilde{\phi}, \omega_{0}\right)$ satisfy

$$
Q_{1 n}(\widetilde{\phi}, \tilde{\lambda})=0, \quad Q_{2 n}(\widetilde{\phi}, \widetilde{\lambda})=0,
$$

where

$$
\begin{gathered}
Q_{1 n}(\phi, \lambda)=\frac{1}{n-u} \sum_{t=u+1}^{n} \frac{m_{t h}\left(\phi, \omega_{0}\right)}{1+\lambda^{\tau} m_{t h}\left(\phi, \omega_{0}\right)}, \\
Q_{2 n}(\phi, \lambda) \\
=\frac{1}{n-u} \sum_{t=u+1}^{n} \frac{1}{1+\lambda^{\tau} m_{t h}\left(\phi, \omega_{0}\right)}\left(\frac{\partial m_{t h}\left(\phi, \omega_{0}\right)}{\partial \phi^{\tau}}\right)^{\tau} \lambda .
\end{gathered}
$$

The following theorem presents the asymptotic distribution of the profile empirical likelihood.

Theorem 2. Under conditions of Proposition 1, as $n \rightarrow \infty$, the random variable $l_{p}\left(\omega_{0}\right)$, with $\tilde{\phi}$ given in Proposition 1 , converges in distribution to $\chi_{m}^{2}$.

If $c$ is chosen such that $P\left(\chi_{m}^{2} \leq c\right)=a$, then Theorem 2 implies that the asymptotic coverage probability of empirical likelihood confidence region $I_{h c}=\left(\omega: l_{p}(\omega) \leq c\right)$ will be $a$; that is, $P\left(\omega_{0} \in I_{h c}\right)=P\left(l_{p}\left(\omega_{0}\right) \leq c\right)=a+o(1)$, as $n \rightarrow \infty$.

\section{Proofs of the Main Results}

In the following, $\|\cdot\|$ denotes the Euclidian norm for a vector or matrix and $C$ denotes a positive constant which may be different at different places. For $t=0, \pm 1, \pm 2, \ldots$, define

$$
U_{t}-\sum_{i=1}^{p} \varphi_{i}^{0} U_{t-i}=\varepsilon_{t}, \quad V_{t}+\sum_{j=1}^{q} \vartheta_{j}^{0} V_{t-j}=\varepsilon_{t} .
$$

Put $Q_{t}=\left(U_{t-1}, \ldots, U_{t-p}, V_{t-1}, \ldots, V_{t-q}\right)^{\tau}, w_{t}=1 /(1+$ $\left.\sum_{k=1}^{\infty} k^{-\alpha}\left|y_{t-k}\right|\right)^{4}$, and the corresponding partial vector for $\phi_{0}$ is denoted by $Q_{1 t}$. Let

$$
\begin{gathered}
\Sigma=E\left(w_{t} Q_{t} Q_{t}^{\tau}\right), \\
\Sigma_{1}=E\left(w_{t} Q_{t} Q_{1 t}^{\tau}\right), \\
\Omega=E\left(w_{t}^{2} Q_{t} Q_{t}^{\tau}\right) .
\end{gathered}
$$

Assumptions A1 and A2 imply that, for $\widetilde{\delta}=\min (\delta, 1)$,

$$
E\left(\sum_{k=1}^{\infty} k^{-\alpha / 2}\left|y_{t-k}\right|\right)^{\tilde{\delta}} \leq \sum_{k=1}^{\infty} k^{-\alpha \tilde{\delta} / 2} E\left|y_{t-k}\right|^{\tilde{\delta}}<\infty .
$$

Hence, $\sum_{k=1}^{\infty} k^{-\alpha / 2}\left|y_{t-k}\right|<\infty$ with probability 1, which ensures that $w_{t}$ is well defined. Note that $\left\|Q_{t}\right\| \leq$ $C \sum_{j=1}^{\infty} r^{j}\left|y_{t-j}\right|$ for some $0<r<1$ and

$$
w_{t}^{1 / 2}\left\|Q_{t}\right\| \leq \frac{C \sum_{j=1}^{\infty} r^{j}\left|y_{t-j}\right|}{1+\sum_{k=1}^{\infty} k^{-\alpha}\left|y_{t-k}\right|} \leq C \sum_{k=1}^{\infty} r^{k} k^{\alpha}<\infty .
$$

Then, $\Sigma, \Sigma_{1}$, and $\Omega$ are well-defined (finite) matrices. For simplicity, we denote $\left(\phi, \omega_{0}\right)$ and $\left(\phi_{0}, \omega_{0}\right)$ by $\phi$ and $\phi_{0}$, respectively, in this section. The following notations will be used in the proofs. Let

$$
\begin{gathered}
Z_{n}(\phi)=\max _{u+1 \leq t \leq n}\left\|m_{t h}(\phi)\right\|, \\
Q_{n h}(\phi)=\frac{1}{n-u} \sum_{t=u+1}^{n} m_{t h}(\phi), \\
S(\phi)=\frac{1}{n-u} \sum_{t=u+1}^{n} m_{t h}(\phi) m_{t h}(\phi)^{\tau} .
\end{gathered}
$$

To prove Proposition 1, we first prove the following lemmas.

Lemma 3. Under the conditions of Proposition 1, as $n \rightarrow \infty$,

$$
\begin{aligned}
& \text { (i) } Q_{n h}\left(\phi_{0}\right)=O\left(\sqrt{\frac{\log n}{n}}\right) \text { a.s., } \\
& \text { (ii) } \frac{\partial Q_{n h}\left(\phi_{0}\right)}{\partial \phi^{\tau}}=-2 f(0) \Sigma_{1}+o(1) \text { a.s. }
\end{aligned}
$$

Proof of Lemma 3. For part (i), we may write

$$
\begin{aligned}
& \sqrt{n-u} Q_{n h}\left(\phi_{0}\right) \\
& =\frac{1}{\sqrt{n-u}} \sum_{t=u+1}^{n} w_{t} Q_{t} G_{h}\left(\varepsilon_{t}\right) \\
& \quad+\frac{1}{\sqrt{n-u}} \sum_{t=u+1}^{n}\left[w_{t} Q_{t}\left(G_{h}\left(\varepsilon_{t}\left(\phi_{0}\right)\right)-G_{h}\left(\varepsilon_{t}\right)\right)\right. \\
& \left.\quad+w_{t}\left(A_{t}\left(\phi_{0}\right)-Q_{t}\right) G_{h}\left(\varepsilon_{t}\left(\phi_{0}\right)\right)\right] \\
& +\frac{1}{\sqrt{n-u}} \sum_{t=u+1}^{n}\left(\widetilde{w}_{t}-w_{t}\right) A_{t}\left(\phi_{0}\right) G_{h}\left(\varepsilon_{t}\left(\phi_{0}\right)\right) \\
& =: K_{1}+K_{2}+K_{3} .
\end{aligned}
$$

For $K_{1}$, we have

$$
\begin{aligned}
\frac{K_{1}}{\sqrt{n-u}}= & \frac{1}{n-u} \sum_{t=u+1}^{n} Z_{t} b_{n t} \\
& +\left(\frac{1}{n-u} \sum_{t=u+1}^{n} Z_{t}\right) O\left(h^{2}\right),
\end{aligned}
$$

where $Z_{t}=w_{t} Q_{t}, b_{n t}=G_{h}\left(\varepsilon_{t}\right)-E\left(G_{h}\left(\varepsilon_{t}\right)\right)$. The second term of $(21)$ is $O(\sqrt{\log n / n})$ a.s. by the ergodicity. Now turning to the first term, we suppose that $Q_{t}$ is the first element $U_{t-1}$ without loss of generality. Note that, for each $n \geq u+1$, $\left\{Z_{t} b_{n t}, \mathscr{F}_{t}, u+1 \leq t \leq n\right\}$ is a sequence of martingale 
differences with $\left|Z_{t} b_{n t}\right| \leq C$, where $\mathscr{F}_{t}=\sigma\left(\varepsilon_{s}, s \leq t\right)$. For some $C_{0}>0$, by the ergodicity, we have

$$
\begin{aligned}
V_{n}^{2} & =\sum_{t=u+1}^{n} E\left\{\left(Z_{t} b_{n t}\right)^{2} \mid \mathscr{F}_{t-1}\right\} \\
& =\sum_{t=u+1}^{n} Z_{t}^{2} E\left(b_{n t}\right)^{2} \\
& \leq C \sum_{t=u+1}^{n} Z_{t}^{2}<C\left(E\left(Z_{t}^{2}\right)+C_{0}\right) n \text { a.s. }
\end{aligned}
$$

Set $y=C\left(E\left(Z_{t}^{2}\right)+C_{0}\right) n$; by Theorem 1.2A in [16], for all $A>0$, we have

$$
\begin{aligned}
P & \left\{\left|\sum_{t=u+1}^{n} Z_{t} b_{n t}\right|>A \sqrt{n \log n}\right\} \\
& =P\left\{\left|\sum_{t=u+1}^{n} Z_{t} b_{n t}\right|>A \sqrt{n \log n}, V_{n}^{2}<y \text { for some } n\right\} \\
& \leq 2 \exp \left\{\frac{-A^{2} n \log n}{2(y+C A \sqrt{n \log n})}\right\} \\
& =2 \exp \left\{\frac{-A^{2} \log n}{2 C\left(E\left(Z_{t}^{2}\right)+C_{0}\right)+2 C A \sqrt{\log n / n}}\right\} .
\end{aligned}
$$

Choosing $A$ such that $A^{2}>2 C\left(E\left(Z_{t}^{2}\right)+C_{0}\right)$, by the BorelCantelli lemma, the first term of (21) is $O(\sqrt{\log n / n})$ a.s. Thus, $K_{1}$ is $O(\sqrt{\log n})$ a.s. For $K_{2}$, by Davis [2], it holds that $\mid \varepsilon_{t}-$ $\varepsilon_{t}\left(\phi_{0}\right) \mid \leq \xi_{t}$, and $\left\|A_{t}\left(\phi_{0}\right)-Q_{t}\right\| \leq \xi_{t}$, where $\xi_{t}=C \sum_{j=t}^{\infty} r^{j}\left|y_{t-j}\right|$ for some $0<r<1$. Therefore,

$$
\begin{aligned}
\left\|K_{2}\right\| \leq & \frac{C}{h \sqrt{n-u}} \sum_{t=u+1}^{n} w_{t}\left\|Q_{t}\right\|\left|\varepsilon_{t}-\varepsilon_{t}\left(\phi_{0}\right)\right| \\
& +\frac{C}{\sqrt{n-u}} \sum_{t=u+1}^{n} w_{t}\left\|Q_{t}-A_{t}\left(\phi_{0}\right)\right\| \\
\leq & \frac{C}{h \sqrt{n-u}} \sum_{t=u+1}^{n} \xi_{t}+\frac{C}{\sqrt{n-u}} \sum_{t=u+1}^{n} \xi_{t} \\
= & \frac{C}{h \sqrt{n-u}} \sum_{t=u+1}^{n} r^{t} \sum_{l=0}^{\infty} r^{l}\left|y_{-l}\right| \\
& +\frac{C}{\sqrt{n-u}} \sum_{t=u+1}^{n} r^{t} \sum_{l=0}^{\infty} r^{l}\left|y_{-l}\right| \stackrel{\text { a.s. }}{\longrightarrow} 0 .
\end{aligned}
$$

Thus, $K_{2}$ is $O(\sqrt{\log n})$ a.s. For $K_{3}$, we have

$$
\begin{aligned}
& \left\|K_{3}\right\| \\
& \quad \leq \frac{C}{\sqrt{n-u}} \sum_{t=u+1}^{n}\left|w_{t}-\widetilde{w}_{t}\right|\left\|A_{t}\left(\phi_{0}\right)\right\| \\
& \quad \leq \frac{C}{\sqrt{n-u}} \sum_{t=u+1}^{n} \frac{\sum_{k=t}^{\infty} k^{-\alpha}\left|y_{t-k}\right| \sum_{j=1}^{t-1} r^{j}\left|y_{t-j}\right|}{1+\sum_{k=1}^{t-1} k^{-\alpha}\left|y_{t-k}\right|}
\end{aligned}
$$

$$
\begin{aligned}
\leq & \frac{C}{\sqrt{n-u}} \sum_{t=u+1}^{n} \sum_{k=t}^{\infty} k^{-\alpha}\left|y_{t-k}\right| \sum_{j=1}^{t-1} r^{j} j^{\alpha} \\
\leq & \frac{C}{\sqrt{n-u}} \sum_{t=u+1}^{n} \sum_{k=t}^{\infty} k^{-\alpha}\left|y_{t-k}\right| \\
\leq & \frac{C}{\sqrt{n-u}} \sum_{t=u+1}^{n} \sum_{l=0}^{\infty}(t+l)^{-\alpha}\left|y_{-l}\right| \\
\leq & \frac{C}{\sqrt{n-u}} \sum_{t=u+1}^{n}\left(t^{-\alpha}\left|y_{0}\right|+\sum_{l=1}^{\infty} 2^{-\alpha} t^{-\alpha / 2} l^{-\alpha / 2}\left|y_{-l}\right|\right) \\
= & \frac{C}{\sqrt{n-u}} \sum_{t=u+1}^{n} t^{-\alpha}\left|y_{0}\right| \\
& +\frac{C}{\sqrt{n-u}} \sum_{t=u+1}^{n} t^{-\alpha / 2} \sum_{l=1}^{\infty} l^{-\alpha / 2}\left|y_{-l}\right| \stackrel{\text { a.s. }}{\longrightarrow} 0
\end{aligned}
$$

because we have the facts that $\left\|A_{t}\left(\phi_{0}\right)\right\| \leq C \sum_{j=1}^{t-1} r^{j}\left|y_{t-j}\right|$ (see [2]) and $(t+l)^{-\alpha} \leq 2^{-\alpha}(t l)^{-\alpha / 2}$ for $t>0, l>0$. Thus, $K_{3}$ is also $O(\sqrt{\log n})$ a.s. Therefore part (i) holds. For the proof of part (ii), we may write

$$
\begin{aligned}
& \frac{\partial Q_{n h}\left(\phi_{0}\right)}{\partial \phi^{\tau}} \\
& =-\frac{1}{(n-u) h} \sum_{t=u+1}^{n} \widetilde{w}_{t} A_{t}\left(\phi_{0}\right) A_{1 t}^{\tau}\left(\phi_{0}\right) \\
& \quad \times\left[K\left(\frac{\varepsilon_{t}\left(\phi_{0}\right)}{h}\right)+K\left(-\frac{\varepsilon_{t}\left(\phi_{0}\right)}{h}\right)\right] \\
& \quad+\frac{1}{n-u} \sum_{t=u+1}^{n} \widetilde{w}_{t} \frac{\partial A_{t}\left(\phi_{0}\right)}{\partial \phi^{\tau}} G_{h}\left(\varepsilon_{t}\left(\phi_{0}\right)\right) \\
& =D_{1}+D_{2},
\end{aligned}
$$

where $A_{1 t}(\phi)=-\partial \varepsilon_{t}(\phi) / \partial \phi$. For $D_{1}$, we may write

$$
\begin{aligned}
D_{1}=-\frac{1}{(n-u) h} \sum_{t=u+1}^{n} w_{t} Q_{t} Q_{1 t}^{\tau}\left(K\left(\frac{\varepsilon_{t}}{h}\right)+K\left(-\frac{\varepsilon_{t}}{h}\right)\right) & -\frac{1}{(n-u) h} \sum_{t=u+1}^{n}\left[\widetilde{w}_{t} A_{t}\left(\phi_{0}\right) A_{1 t}^{\tau}\left(\phi_{0}\right)\right. \\
& \times\left(K\left(\frac{\varepsilon_{t}\left(\phi_{0}\right)}{h}\right)+K\left(-\frac{\varepsilon_{t}\left(\phi_{0}\right)}{h}\right)\right) \\
& \left.-w_{t} Q_{t} Q_{1 t}^{\tau}\left(K\left(\frac{\varepsilon_{t}}{h}\right)+K\left(-\frac{\varepsilon_{t}}{h}\right)\right)\right] \\
= &
\end{aligned}
$$


Note that

$$
\begin{aligned}
D_{11}= & -\frac{1}{(n-u) h} \sum_{t=u+1}^{n} T_{t} c_{n t}-\frac{1}{(n-u) h} \\
& \times \sum_{t=u+1}^{n} w_{t} Q_{t} Q_{1 t}^{\tau} E\left(K\left(\frac{\varepsilon_{t}}{h}\right)+K\left(-\frac{\varepsilon_{t}}{h}\right)\right) \\
= & -\frac{1}{(n-u) h} \sum_{t=u+1}^{n} T_{t} c_{n t} \\
& -\left[\frac{1}{n-u} \sum_{t=u+1}^{n} w_{t} Q_{t} Q_{1 t}^{\tau}\right](2 f(0)+o(h)),
\end{aligned}
$$

where $T_{t}=w_{t} Q_{t} Q_{1 t}^{\tau}$ and $c_{n t}=K\left(\varepsilon_{t} / h\right)+K\left(-\varepsilon_{t} / h\right)-E\left[K\left(\varepsilon_{t} /\right.\right.$ $\left.h)+K\left(-\varepsilon_{t} / h\right)\right]$. The second term of $(28)$ is $-2 f(0) \Sigma_{1}$ a.s. by the ergodicity. We will prove that the first term is $o(1)$ a.s. We suppose that $Q_{t}$ is the first element $U_{t-1}$ without loss of generality. Note that, for each $n \geq u+1,\left\{T_{t} c_{n t}, \mathscr{F}_{t}, u+1 \leq\right.$ $t \leq n\}$ is a sequence of martingale differences with $\left|T_{t} c_{n t}\right| \leq C$, and

$$
\begin{aligned}
V_{n}^{2} & =\sum_{t=u+1}^{n} E\left\{\left(T_{t} c_{n t}\right)^{2} \mid \mathscr{F}_{t-1}\right\} \\
& =\sum_{t=u+1}^{n} T_{t}^{2} E\left(c_{n t}\right)^{2}<n C\left(f(0) C_{0} h+O\left(h^{2}\right)\right) \text { a.s., }
\end{aligned}
$$

where $C_{0}>0$ is a constant. Set $y=n C\left(f(0) C_{0} h+O\left(h^{2}\right)\right)$; by Theorem 1.2A in [16], for all $\varepsilon>0$, we have

$$
\begin{aligned}
P & \left\{\left|\sum_{t=u+1}^{n} T_{t} c_{n t}\right|>(n h) \varepsilon\right\} \\
& =P\left\{\left|\sum_{t=u+1}^{n} T_{t} c_{n t}\right|>(n h) \varepsilon, V_{n}^{2}<y \text { for some } n\right\} \\
& \leq 2 \exp \left\{\frac{-(n h)^{2} \varepsilon^{2}}{2(y+C n h \varepsilon)}\right\} \\
& =2 \exp \left\{\frac{-n h \varepsilon^{2}}{2 C\left(f(0) C_{0}+O(h)\right)+2 C \varepsilon}\right\} .
\end{aligned}
$$

The result follows from the Borel-Cantelli lemma. Thus $D_{11}=$ $-2 f(0) \Sigma_{1}+o(1)$ a.s. Similar to $K_{2}$ and $K_{3}$, we have

$$
\begin{aligned}
& \left\|D_{12}\right\| \\
& \begin{array}{l}
\leq \frac{1}{(n-u) h} \sum_{t=u+1}^{n} w_{t}\left(\left\|Q_{t}-A_{t}\left(\phi_{0}\right)\right\|\left\|Q_{1 t}\right\|\right. \\
\left.\quad+\left\|A_{t}\left(\phi_{0}\right)\right\|\left\|Q_{1 t}-A_{1 t}\left(\phi_{0}\right)\right\|\right) \\
\quad\left|K\left(\frac{\varepsilon_{t}\left(\phi_{0}\right)}{h}\right)+K\left(-\frac{\varepsilon_{t}\left(\phi_{0}\right)}{h}\right)\right|
\end{array}
\end{aligned}
$$

$$
\begin{aligned}
& +\frac{1}{(n-u) h_{t=u+1}}\left|w_{t}-\widetilde{w}_{t}\right|\left\|A_{t}\left(\phi_{0}\right)\right\|\left\|A_{1 t}\left(\phi_{0}\right)\right\| \\
& \times\left|K\left(\frac{\varepsilon_{t}\left(\phi_{0}\right)}{h}\right)+K\left(-\frac{\varepsilon_{t}\left(\phi_{0}\right)}{h}\right)\right| \\
& +\frac{1}{(n-u) h} \sum_{t=u+1}^{n} w_{t}\left\|Q_{t}\right\|\left\|Q_{1 t}\right\| \\
& \times \mid\left[K\left(\frac{\varepsilon_{t}}{h}\right)+K\left(-\frac{\varepsilon_{t}}{h}\right)\right] \\
& \leq \frac{C}{(n-u) h_{t=u+1}} \sum_{t}^{n} w_{t}\left(\left\|Q_{t}-A_{t}\left(\phi_{0}\right)\right\|\left\|Q_{1 t}\right\|\right. \\
& +\frac{C}{(n-u) h^{2}} \sum_{t=u+1}^{n} \xi_{t} \stackrel{\text { a.s. }}{\longrightarrow} 0 . \\
& \left.+\frac{C}{(n-u) h} \sum_{t=u+1}^{n} \mid \phi_{0}\right) \\
& +\frac{C}{(n-u) h_{t=u+1}} \sum_{t}^{n} \xi_{t}+\frac{C}{(n-u) h_{t}^{2}} \sum_{t=u+1}^{n} \sum_{t=u+1}^{n} \sum_{k=t}^{\infty} k^{-\alpha}\left|y_{t-k}\right| \\
& +Q_{t}\|\| Q_{t}\left(\phi_{0}\right)\|\| \phi_{1 t}\left(\phi_{0}\right) \| \mid \\
& +\varepsilon_{t}-\varepsilon_{t}\left(\phi_{0}\right) \mid
\end{aligned}
$$

Therefore, $D_{1}=-2 f(0) \Sigma_{1}+o(1)$ a.s. For $D_{2}$, from the definition of $A_{t}(\phi)$, it holds for $t>\max (p, q)$ that

$$
\theta(B) \frac{\partial A_{t, i}(\phi)}{\partial \phi_{j}}=0, \quad i, j=1, \ldots, p
$$

$$
\begin{gathered}
\theta(B) \frac{\partial A_{t, i}(\phi)}{\partial \phi_{j+p}}=-A_{t-j, i}(\phi), \quad i=1, \ldots, p, j=1, \ldots, q, \\
\theta(B) \frac{\partial A_{t, j+p}(\phi)}{\partial \phi_{i}}=-A_{t-j, i}(\phi), \quad i=1, \ldots, p, j=1, \ldots, q, \\
\theta(B) \frac{\partial A_{t, i+p}(\phi)}{\partial \phi_{j+p}}=-A_{t-j, i+p}(\phi)-A_{t-i, j+p}(\phi), \\
i, j=1, \ldots, q,
\end{gathered}
$$


where $B$ is the backshift operator. For $t=0, \pm 1, \pm 2, \ldots$, define

$$
\begin{gathered}
\theta_{0}(B) X_{t(i, j)}=0, \quad i, j=1, \ldots, p, \\
\theta_{0}(B) X_{t(i, j+p)}=-Q_{t-j, i}, \quad i=1, \ldots, p, j=1, \ldots, q, \\
\theta_{0}(B) X_{t(j+p, i)}=-Q_{t-j, i}, \quad i=1, \ldots, p, j=1, \ldots, q, \\
\theta_{0}(B) X_{t(i+p, j+p)}=-Q_{t-j, i+p}-Q_{t-i, j+p}, \\
i, j=1, \ldots, q,
\end{gathered}
$$

where $Q_{t, i}$ is the $i$ th component of $Q_{t}$. Put $X_{t}=\left(X_{t(i, j)}\right)$; similar to [13], we have that $\left\|\partial A_{t}\left(\phi_{0}\right) / \partial \phi^{\tau}\right\| \leq C \sum_{j=1}^{t-1} r^{j}\left|y_{t-j}\right|$, $\left\|X_{t}\right\| \leq C \sum_{j=1}^{\infty} r^{j}\left|y_{t-j}\right|$, and $\left\|X_{t}-\partial A_{t}\left(\phi_{0}\right) / \partial \phi^{\tau}\right\| \leq \xi_{t}$. Then, we may write

$$
\begin{aligned}
D_{2}= & \frac{1}{n-u} \sum_{t=u+1}^{n} w_{t} X_{t} G_{h}\left(\varepsilon_{t}\right) \\
& +\frac{1}{n-u} \sum_{t=u+1}^{n}\left[\widetilde{w}_{t} \frac{\partial A_{t}\left(\phi_{0}\right)}{\partial \phi^{\tau}} G_{h}\left(\varepsilon_{t}\left(\phi_{0}\right)\right)-w_{t} X_{t} G_{h}\left(\varepsilon_{t}\right)\right] \\
= & D_{21}+D_{22} .
\end{aligned}
$$

Similar to $D_{11}$ and $D_{12}$, we have that $D_{21}$ and $D_{22}$ are $o(1)$ a.s. This completes the proof.

Lemma 4. Under the conditions of Proposition 1 , as $n \rightarrow \infty$,

$$
\begin{aligned}
& \text { (i) } \quad Z_{n}(\phi)=o\left(n^{1 / 3}\right) \text { a.s. } \\
& \text { (ii) } S(\phi)=\Omega+o(1) \text { a.s. }
\end{aligned}
$$

hold uniformly in $B$.

Proof of Lemma 4. For part (i), from [2], we have that $\left\|\partial A_{t}(\phi) / \partial \phi^{\tau}\right\| \leq C \sum_{j=1}^{t-1} r^{j}\left|y_{t-j}\right|$ and $\left\|A_{t}(\phi)\right\| \leq$ $C \sum_{j=1}^{t-1} r^{j}\left|y_{t-j}\right|$ uniformly hold in the ball $B$ for sufficiently large $n$. Then, for each $\phi \in B$, we have

$$
\begin{aligned}
\left\|m_{t h}(\phi)\right\| & \leq \widetilde{w}_{t}\left\|A_{t}(\phi)\right\| \\
& \leq \frac{C \sum_{j=1}^{t-1} r^{j}\left|y_{t-j}\right|}{1+\sum_{k=1}^{t-1} k^{-\alpha}\left|y_{t-k}\right|} \leq C \sum_{k=1}^{t-1} r^{k} k^{\alpha} \\
& \leq C \sum_{k=1}^{\infty} r^{k} k^{\alpha}<\infty .
\end{aligned}
$$

Thus, part (i) holds. For part (ii), similar to the proof of Lemma 3, we have that $S\left(\phi_{0}\right)=\Omega+o(1)$ a.s. For each $\phi \in B$, by Taylor expansion, we have

$$
\begin{aligned}
S(\phi) & -S\left(\phi_{0}\right) \\
= & \frac{1}{n-u} \sum_{t=u+1}^{n} m_{t h}\left(\phi_{0}\right)\left\{\frac{\partial m_{t h}\left(\phi^{*}\right)}{\partial \phi^{\tau}}\left(\phi-\phi_{0}\right)\right\}^{\tau} \\
& +\frac{1}{n-u} \sum_{t=u+1}^{n}\left\{\frac{\partial m_{t h}\left(\phi^{*}\right)}{\partial \phi^{\tau}}\left(\phi-\phi_{0}\right)\right\} m_{t h}^{\tau}\left(\phi_{0}\right)
\end{aligned}
$$

$$
\begin{aligned}
& +\frac{1}{n-u} \sum_{t=u+1}^{n}\left\{\frac{\partial m_{t h}\left(\phi^{*}\right)}{\partial \phi^{\tau}}\left(\phi-\phi_{0}\right)\right\} \\
& \quad \times\left\{\frac{\partial m_{t h}\left(\phi^{*}\right)}{\partial \phi^{\tau}}\left(\phi-\phi_{0}\right)\right\}^{\tau} \\
& =: T_{1}+T_{2}+T_{3},
\end{aligned}
$$

where $\phi^{*}$ lies between $\phi_{0}$ and $\phi$. For $T_{1}$, we have

$$
\begin{aligned}
\left\|T_{1}\right\| \leq & \frac{1}{n-u} \sum_{t=u+1}^{n} \widetilde{w}_{t}^{2}\left\|A_{t}\left(\phi_{0}\right)\right\|\left\|\frac{\partial A_{t}\left(\phi^{*}\right)}{\partial \phi^{\tau}}\right\|\left\|\phi-\phi_{0}\right\| \\
& +\frac{1}{(n-u) h} \sum_{t=u+1}^{n} \widetilde{w}_{t}^{2}\left\|A_{t}\left(\phi_{0}\right)\right\|\left\|A_{t}\left(\phi^{*}\right)\right\| \\
& \times\left\|A_{1 t}\left(\phi^{*}\right)\right\|\left\|\phi-\phi_{0}\right\| \\
\leq & \left.\frac{C d_{n}}{n-u} \sum_{t=u+1}^{n}\left(\sum_{j=1}^{t-1} r^{j} j^{\alpha}\right)^{2} \frac{\varepsilon_{t}\left(\phi^{*}\right)}{h}\right)+K\left(-\frac{\varepsilon_{t}\left(\phi^{*}\right)}{h}\right) \mid \\
& +\frac{C d_{n}}{(n-u) h} \sum_{t=u+1}^{n}\left(\sum_{j=1}^{t-1} r^{j} j^{\alpha} \stackrel{\text { a.s. }}{\longrightarrow} 0 .\right.
\end{aligned}
$$

Similarly, we have $T_{2} \stackrel{\text { a.s. }}{\longrightarrow} 0$ and $T_{3} \stackrel{\text { a.s. }}{\longrightarrow} 0$. This completes the proof.

Proof of Proposition 1. For $\phi \in B$, by Taylor expansion,

$$
\begin{aligned}
Q_{n h}(\phi)= & Q_{n h}\left(\phi_{0}\right)+\frac{\partial Q_{n h}\left(\phi_{0}\right)}{\partial \phi^{\tau}}\left(\phi-\phi_{0}\right) \\
& +\frac{1}{2} \sum_{j, k=1}^{p+q-m} \frac{\partial^{2} Q_{n h}\left(\phi_{0}\right)}{\partial \phi_{j} \partial \phi_{k}}\left(\phi_{j}-\phi_{j}^{0}\right)\left(\phi_{k}-\phi_{k}^{0}\right) \\
& +\frac{1}{6} \sum_{j, k, l=1}^{p+q-m} \frac{\partial^{3} Q_{n h}\left(\phi_{*}\right)}{\partial \phi_{j} \partial \phi_{k} \partial \phi_{l}} \\
& \times\left(\phi_{j}-\phi_{j}^{0}\right)\left(\phi_{k}-\phi_{k}^{0}\right)\left(\phi_{l}-\phi_{l}^{0}\right),
\end{aligned}
$$

where $\phi_{*}$ lies between $\phi_{0}$ and $\phi$. Note that the final term on the right side of (39) can be written as

$$
\begin{aligned}
& \frac{1}{6} \sum_{j, k, l=1}^{p+q-m}\left[\frac{\left(\phi_{j}-\phi_{j}^{0}\right)\left(\phi_{k}-\phi_{k}^{0}\right)\left(\phi_{l}-\phi_{l}^{0}\right)}{h^{3}}\right] \\
& \quad \times\left(\frac{h^{3}}{n-u} \sum_{t=u+1}^{n} \frac{\partial^{3} m_{t h}\left(\phi_{*}\right)}{\partial \phi_{j} \partial \phi_{k} \partial \phi_{l}}\right),
\end{aligned}
$$


which is $o\left(\delta_{n}\right)$ a.s., where $\delta_{n}=\left\|\phi-\phi_{0}\right\|$, because $d_{n}^{2} / h^{3}=$ $1 / n^{2 \beta-3 \gamma} \rightarrow 0$ and

$$
\left\|\frac{h^{3}}{n-u} \sum_{t=u+1}^{n} \frac{\partial^{3} m_{t h}\left(\phi_{*}\right)}{\partial \phi_{j} \partial \phi_{k} \partial \phi_{l}}\right\| \leq C
$$

The third term on the right side of (39) can be written as

$$
\begin{aligned}
\frac{1}{2} \sum_{j, k=1}^{p+q-m} & {\left[\frac{\left(\phi_{j}-\phi_{j}^{0}\right)\left(\phi_{k}-\phi_{k}^{0}\right)}{h}\right] } \\
& \times\left\{\frac{h}{n-u} \sum_{t=u+1}^{n} \frac{\partial^{2} m_{t h}\left(\phi_{0}\right)}{\partial \phi_{j} \partial \phi_{k}}\right\},
\end{aligned}
$$

which is also $o\left(\delta_{n}\right)$ a.s., because $d_{n} / h=1 / n^{\beta-\gamma} \rightarrow 0$, and

$$
\frac{h}{n-u} \sum_{t=u+1}^{n} \frac{\partial^{2} m_{t h}\left(\phi_{0}\right)}{\partial \phi_{j} \partial \phi_{k}}=o(1) \text { a.s., }
$$

by a similar proof of Lemma 3. Therefore,

$$
Q_{n h}(\phi)=Q_{n h}\left(\phi_{0}\right)+\frac{\partial Q_{n h}\left(\phi_{0}\right)}{\partial \phi^{\tau}}\left(\phi-\phi_{0}\right)+o\left(\delta_{n}\right) \text { a.s., }
$$

uniformly about $\phi \in B$. Denote $\phi=\phi_{0}+\mu d_{n}$, for $\phi \in\{\phi$ : $\left.\left\|\phi-\phi_{0}\right\|=d_{n}\right\}$, where $\|\mu\|=1$. Now, we give a lower bound for $l_{h}(\phi)$ on the surface of the ball. Similar to [6], by Lemmas 3 and 4 , we have

$$
\begin{aligned}
& l_{h}(\phi) \\
& =(n-u) Q_{n h}(\phi)^{\tau} S(\phi)^{-1} Q_{n h}(\phi)+o\left(n^{4 / 3-3 \beta}\right) \text { a.s. } \\
& =(n-u)\left[O\left(\sqrt{\frac{\log n}{n}}\right)\right. \\
& \left.\quad+(-2 f(0)) \Sigma_{1} \mu d_{n}+o\left(d_{n}\right)\right]^{\tau} \Omega^{-1} \\
& \quad \times\left[O\left(\sqrt{\frac{\log n}{n}}\right)+(-2 f(0)) \Sigma_{1} \mu d_{n}+o\left(d_{n}\right)\right] \\
& \quad+o\left(n^{4 / 3-3 \beta}\right) \text { a.s. } \\
& \geq(c-\varepsilon) n^{1-2 \beta} \text { a.s., }
\end{aligned}
$$

where $c-\varepsilon>0$ and $c$ is the smallest eigenvalue of $4 f^{2}(0) \Sigma_{1}^{\tau} \Omega^{-1} \Sigma_{1}$. Similarly,

$$
\begin{aligned}
& l_{h}\left(\phi_{0}\right) \\
& \quad=(n-u) Q_{n h}\left(\phi_{0}\right)^{\tau} S\left(\phi_{0}\right)^{-1} Q_{n h}\left(\phi_{0}\right)+o(1) \text { a.s. } \\
& \quad=O(\log n) \text { a.s. }
\end{aligned}
$$

Since $l_{h}(\phi)$ is a continuous function about $\phi$ as $\phi$ belongs to the ball $B, l_{h}(\phi)$ attains its minimum value at some point $\tilde{\phi}$ in the interior of this ball, and $\widetilde{\phi}$ satisfies $\partial l_{h}(\widetilde{\phi}) / \partial \phi=0$, it follows that (12) holds. This completes the proof.

Proof of Theorem 2. Similar to the proof of Theorem 2 of Qin and Lawless [17], we have

$$
\left(\begin{array}{c}
\tilde{\lambda} \\
\tilde{\phi}-\phi_{0}
\end{array}\right)=S_{n}^{-1}\left(\begin{array}{c}
-Q_{1 n}\left(\phi_{0}, 0\right)+o_{p}\left(n^{-1 / 2}\right) \\
o_{p}\left(n^{-1 / 2}\right)
\end{array}\right),
$$

where

$$
\begin{aligned}
S_{n} & =\left(\begin{array}{cc}
\frac{\partial Q_{1 n}\left(\phi_{0}, 0\right)}{\partial \lambda^{\tau}} & \frac{\partial Q_{1 n}\left(\phi_{0}, 0\right)}{\partial \phi^{\tau}} \\
\frac{\partial Q_{2 n}\left(\phi_{0}, 0\right)}{\partial \lambda^{\tau}} & 0
\end{array}\right) \stackrel{p}{\longrightarrow}\left(\begin{array}{cc}
S_{11} & S_{12} \\
S_{21} & 0
\end{array}\right) \\
& =\left(\begin{array}{cc}
-\Omega & -2 f(0) \Sigma_{1} \\
-2 f(0) \Sigma_{1}^{\tau} & 0
\end{array}\right) .
\end{aligned}
$$

By the standard arguments in the proof of empirical likelihood (see [6]), we have

$$
\begin{aligned}
l_{p}\left(\omega_{0}\right) & \\
= & -(n-u)\left(Q_{n h}\left(\phi_{0}\right)^{\tau}, 0\right) S_{n}^{-1}\left(Q_{n h}\left(\phi_{0}\right)^{\tau}, 0\right)^{\tau}+o_{p}(1) \\
= & \left(\Omega^{-1 / 2} \sqrt{n-u} Q_{n h}\left(\phi_{0}\right)\right)^{\tau} \\
& \times\left(I-4 f^{2}(0) \Omega^{-1 / 2} \Sigma_{1} \Delta^{-1} \Sigma_{1}^{\tau} \Omega^{-1 / 2}\right) \\
& \times\left(\Omega^{-1 / 2} \sqrt{n-u} Q_{n h}\left(\phi_{0}\right)\right)+o_{p}(1),
\end{aligned}
$$

where $\Delta=4 f^{2}(0) \Sigma_{1}^{\tau} \Omega^{-1} \Sigma_{1}$. Since $\sqrt{n-u} Q_{n h}\left(\phi_{0}\right) \stackrel{d}{\rightarrow} N(0, \Omega)$ and

$$
\begin{aligned}
\operatorname{tr} & \left\{4 f^{2}(0) \Omega^{-1 / 2} \Sigma_{1} \Delta^{-1} \Sigma_{1}^{\tau} \Omega^{-1 / 2}\right\} \\
& =\operatorname{tr}\left\{\Delta^{-1} 4 f^{2}(0) \Sigma_{1}^{\tau} \Omega^{-1} \Sigma_{1}\right\}=p+q-m,
\end{aligned}
$$

it follows that $l_{p}\left(\omega_{0}\right) \stackrel{d}{\rightarrow} \chi_{m}^{2}$.

\section{Simulation Studies}

We generated data from a simple $\operatorname{ARMA}(1,1)$ model $y_{t}=$ $\varphi_{1} y_{t-1}+\varepsilon_{t}+\vartheta_{1} \varepsilon_{t-1}$, with $N(0,1), t_{2}$, and Cauchy innovation distribution. We set $u=20, \alpha=3$, and the true value $\left(\varphi_{1}, \vartheta_{1}\right)=(0.4,0.7)$ or $(-0.5,0.7)$, where $\varphi_{1}$ is the parameter of interest. The sample size $n=50,100,150,200$, and 2,000 replications are conducted in all cases. We smooth the estimating equations using kernel

$$
K(x)=\frac{1}{\sqrt{2 \pi} \sigma} e^{-x^{2} / 2 \sigma^{2}},
$$


TABLE 1: The coverage probability of confidence intervals when $\varepsilon_{t} \sim N(0,1)$.

\begin{tabular}{|c|c|c|c|c|c|c|}
\hline & $n$ & $\mathrm{EL}(0.27)$ & EL(0.30) & $\mathrm{EL}(0.32)$ & $\mathrm{NA}(0.25)$ & $\mathrm{NA}(0.20)$ \\
\hline \multirow{4}{*}{$\begin{array}{l}a=0.9 \\
\varphi_{1}=0.4\end{array}$} & 50 & 0.8818 & 0.8820 & 0.8822 & 0.8193 & 0.7875 \\
\hline & 100 & 0.8898 & 0.8896 & 0.8897 & 0.8655 & 0.8431 \\
\hline & 150 & 0.8926 & 0.8927 & 0.8932 & 0.8692 & 0.8395 \\
\hline & 200 & 0.8983 & 0.8983 & 0.8986 & 0.8666 & 0.8363 \\
\hline \multirow{4}{*}{$\begin{array}{l}a=0.9 \\
\varphi_{1}=-0.5\end{array}$} & 50 & 0.8888 & 0.8885 & 0.8892 & 0.8156 & 0.7813 \\
\hline & 100 & 0.8967 & 0.8968 & 0.8973 & 0.8738 & 0.8448 \\
\hline & 150 & 0.8943 & 0.8944 & 0.8946 & 0.8823 & 0.8574 \\
\hline & 200 & 0.8972 & 0.8979 & 0.8977 & 0.8931 & 0.8692 \\
\hline \multirow{4}{*}{$\begin{array}{l}a=0.95 \\
\varphi_{1}=0.4\end{array}$} & 50 & 0.9347 & 0.9350 & 0.9350 & 0.8724 & 0.8425 \\
\hline & 100 & 0.9424 & 0.9430 & 0.9431 & 0.9123 & 0.8862 \\
\hline & 150 & 0.9467 & 0.9471 & 0.9470 & 0.9157 & 0.8936 \\
\hline & 200 & 0.9494 & 0.9494 & 0.9497 & 0.9160 & 0.8937 \\
\hline \multirow{4}{*}{$\begin{array}{l}a=0.95 \\
\varphi_{1}=-0.5\end{array}$} & 50 & 0.9404 & 0.9404 & 0.9404 & 0.8705 & 0.8407 \\
\hline & 100 & 0.9472 & 0.9474 & 0.9474 & 0.9134 & 0.8931 \\
\hline & 150 & 0.9481 & 0.9479 & 0.9476 & 0.9248 & 0.9052 \\
\hline & 200 & 0.9495 & 0.9495 & 0.9490 & 0.9326 & 0.9152 \\
\hline
\end{tabular}

where $\sigma=0.1$, which is the so-called Gaussian kernel. The coverage probabilities of smoothed empirical likelihood confidence regions $I_{h c}$ with the bandwidth $h=1 / n^{\gamma}$ are denoted by $\operatorname{EL}(\gamma)$, where $\gamma=0.27,0.30,0.32$, respectively.

As another benchmark of the simulation experiments, we consider the confidence regions based on the asymptotic normal distribution of WLADE proposed by [3]. To construct the confidence regions, we need to estimate $f(0), \Sigma$, and $\Omega$. We can estimate $f(0)$ by

$$
\widehat{f}(0)=\frac{1}{\widehat{\sigma}_{w} b_{n}(n-u)} \sum_{t=u+1}^{n} \widetilde{w_{t}} \widetilde{K}\left\{\frac{\varepsilon_{t}(\widehat{\theta})}{b_{n}}\right\}
$$

where $\widetilde{K}(x)=\exp (-x) /(1+\exp (-x))^{2}$ is a kernel function on $R$ and $b_{n}=1 / n^{\nu}$ is a bandwidth, $\widehat{\sigma}_{w}=(n-u)^{-1} \sum_{t=u+1}^{n} \widetilde{w}_{t} . \Sigma$ and $\Omega$ can be estimated, respectively, by

$$
\begin{aligned}
& \widehat{\Sigma}=\frac{1}{n-u} \sum_{t=u+1}^{n} \widetilde{w}_{t} \widehat{Q}_{t} \widehat{Q}_{t}^{\tau}, \\
& \widehat{\Omega}=\frac{1}{n-u} \sum_{t=u+1}^{n} \widetilde{w}_{t}^{2} \widehat{Q}_{t} \widehat{Q}_{t}^{\tau},
\end{aligned}
$$

where $\widehat{Q}_{t}$ is defined in the same manner as $Q_{t}, \theta_{0}$ is replaced by $\hat{\theta}$, and $\varepsilon_{t}$ is replaced by $\varepsilon_{t}(\hat{\theta})$; see (14). Based on this, we can construct a NA confidence region (i.e., based on the normal approximation of WLADE). The coverage probabilities of confidence regions $I_{\mathrm{NA}}$ based on the bandwidth $b_{n}=1 / n^{\nu}$ are denoted by NA $(\nu)$, with $\nu=0.25,0.20$, respectively. Tables 1,2 , and 3 show the probabilities of the confidence intervals of $\varphi_{1}$ at confidence levels 0.9 and 0.95 , respectively.

The simulation results can be summarized as follows. The coverage probabilities of $\mathrm{NA}(\nu)$ are much smaller than the nominal levels and very sensitive to the choice of bandwidth $b_{n}$ and $\varepsilon_{t}$. On the other hand, the coverage probabilities of $\operatorname{EL}(\gamma)$ are much better and less sensitive to the choice of bandwidth $h$ and $\varepsilon_{t}$. As the sample size $n$ increases, the coverage probabilities for both increase to the nominal levels, as one might expect.

\section{Conclusions}

This paper explores a profile empirical likelihood method to construct confidence regions for the partial parameters of interest in IVARMA models. We started with the foundation of estimating equations of WLADE; then from there, we derived smoothed empirical likelihood. Moreover, we have proved that the resulting statistics has asymptotic standard chi-squared distribution. Hence there is no need to estimate any additional quantity such as the asymptotic variance. The simulations indeed show that the proposed method has a good finite sample behavior, which experimentally confirms our method.

\section{Conflict of Interests}

The authors declare that they have no conflict of interests. 
TABLE 2: The coverage probability of confidence intervals when $\varepsilon_{t} \sim t_{2}$.

\begin{tabular}{|c|c|c|c|c|c|c|}
\hline & $n$ & $\mathrm{EL}(0.27)$ & $\mathrm{EL}(0.30)$ & $\operatorname{EL}(0.32)$ & $\mathrm{NA}(0.25)$ & $\mathrm{NA}(0.20)$ \\
\hline \multirow{4}{*}{$\begin{array}{l}a=0.9 \\
\varphi_{1}=0.4\end{array}$} & 50 & 0.8774 & 0.8776 & 0.8779 & 0.7627 & 0.7290 \\
\hline & 100 & 0.8850 & 0.8849 & 0.8856 & 0.8323 & 0.7995 \\
\hline & 150 & 0.8919 & 0.8917 & 0.8924 & 0.8467 & 0.8157 \\
\hline & 200 & 0.8935 & 0.8932 & 0.8932 & 0.8510 & 0.8229 \\
\hline \multirow{4}{*}{$\begin{array}{l}a=0.9 \\
\varphi_{1}=-0.5\end{array}$} & 50 & 0.8902 & 0.8902 & 0.8899 & 0.7295 & 0.7055 \\
\hline & 100 & 0.8942 & 0.8944 & 0.8950 & 0.8151 & 0.7836 \\
\hline & 150 & 0.8946 & 0.8941 & 0.8937 & 0.8473 & 0.8204 \\
\hline & 200 & 0.8965 & 0.8963 & 0.8961 & 0.8641 & 0.8392 \\
\hline \multirow{4}{*}{$\begin{array}{l}a=0.95 \\
\varphi_{1}=0.4\end{array}$} & 50 & 0.9331 & 0.9327 & 0.9327 & 0.8163 & 0.7854 \\
\hline & 100 & 0.9412 & 0.9412 & 0.9413 & 0.8834 & 0.8542 \\
\hline & 150 & 0.9447 & 0.9446 & 0.9447 & 0.8965 & 0.8718 \\
\hline & 200 & 0.9456 & 0.9458 & 0.9459 & 0.9001 & 0.8762 \\
\hline \multirow{4}{*}{$\begin{array}{l}a=0.95 \\
\varphi_{1}=-0.5\end{array}$} & 50 & 0.9421 & 0.9418 & 0.9418 & 0.7963 & 0.7658 \\
\hline & 100 & 0.9455 & 0.9462 & 0.9460 & 0.8695 & 0.8430 \\
\hline & 150 & 0.9444 & 0.9442 & 0.9440 & 0.8910 & 0.8695 \\
\hline & 200 & 0.9464 & 0.9463 & 0.9464 & 0.9072 & 0.8863 \\
\hline
\end{tabular}

TABLE 3: The coverage probability of confidence intervals when $\varepsilon_{t} \sim$ Cauchy.

\begin{tabular}{|c|c|c|c|c|c|c|}
\hline & $n$ & $\mathrm{EL}(0.27)$ & $\mathrm{EL}(0.30)$ & $\mathrm{EL}(0.32)$ & $\mathrm{NA}(0.25)$ & $\mathrm{NA}(0.20)$ \\
\hline \multirow{4}{*}{$\begin{array}{l}a=0.9 \\
\varphi_{1}=0.4\end{array}$} & 50 & 0.8360 & 0.8358 & 0.8361 & 0.6345 & 0.6018 \\
\hline & 100 & 0.8708 & 0.8708 & 0.8702 & 0.7286 & 0.6942 \\
\hline & 150 & 0.8811 & 0.8819 & 0.8823 & 0.7614 & 0.7270 \\
\hline & 200 & 0.8870 & 0.8864 & 0.8868 & 0.7800 & 0.7467 \\
\hline \multirow{4}{*}{$\begin{array}{l}a=0.9 \\
\varphi_{1}=-0.5\end{array}$} & 50 & 0.8673 & 0.8679 & 0.8678 & 0.5818 & 0.5502 \\
\hline & 100 & 0.8869 & 0.8870 & 0.8874 & 0.6756 & 0.6440 \\
\hline & 150 & 0.8972 & 0.8974 & 0.8974 & 0.7232 & 0.6867 \\
\hline & 200 & 0.8956 & 0.8955 & 0.8953 & 0.7510 & 0.7156 \\
\hline \multirow{4}{*}{$\begin{array}{l}a=0.95 \\
\varphi_{1}=0.4\end{array}$} & 50 & 0.8992 & 0.8994 & 0.8996 & 0.6888 & 0.6557 \\
\hline & 100 & 0.9263 & 0.9261 & 0.9263 & 0.7870 & 0.7530 \\
\hline & 150 & 0.9362 & 0.9366 & 0.9367 & 0.8176 & 0.7857 \\
\hline & 200 & 0.9402 & 0.9398 & 0.9399 & 0.8350 & 0.8044 \\
\hline \multirow{4}{*}{$\begin{array}{l}a=0.95 \\
\varphi_{1}=-0.5\end{array}$} & 50 & 0.9235 & 0.9239 & 0.9240 & 0.6378 & 0.6065 \\
\hline & 100 & 0.9422 & 0.9420 & 0.9420 & 0.7342 & 0.7030 \\
\hline & 150 & 0.9474 & 0.9479 & 0.9481 & 0.7820 & 0.7530 \\
\hline & 200 & 0.9467 & 0.9471 & 0.9470 & 0.8057 & 0.7778 \\
\hline
\end{tabular}

\section{Acknowledgments}

Li's research is partially supported by the Fundamental Research Funds for the Central Universities (no. 2013XK03). He's work is partially supported by the National Natural Science Foundation of China (nos. 11171230 and 11231010).

\section{References}

[1] T. Mikosch, T. Gadrich, C. Kluppelberg, and R. J. Adler, "Parameter estimation for ARMA models with infinite variance innovations," The Annals of Statistics, vol. 23, no. 1, pp. 305-326, 1995.
[2] R. A. Davis, "Gauss-Newton and M-estimation for ARMA processes with infinite variance," Stochastic Processes and their Applications, vol. 63, no. 1, pp. 75-95, 1996.

[3] J. Pan, H. Wang, and Q. Yao, "Weighted least absolute deviations estimation for ARMA models with infinite variance," Econometric Theory, vol. 23, no. 5, pp. 852-879, 2007.

[4] K. Zhu and S. Ling, "The global LAD estimators for finite/ infinite variance $\operatorname{ARMA}(p, q)$ models," Econometric Theory, vol. 28, no. 5, pp. 1065-1086, 2012.

[5] A. B. Owen, "Empirical likelihood ratio confidence intervals for a single functional," Biometrika, vol. 75, no. 2, pp. 237-249, 1988.

[6] A. B. Owen, "Empirical likelihood ratio confidence regions," The Annals of Statistics, vol. 18, no. 1, pp. 90-120, 1990. 
[7] A. B. Owen, Empirical Likelihood, Chapman and Hall, London, UK, 2001.

[8] A. C. Monti, "Empirical likelihood confidence regions in time series models," Biometrika, vol. 84, no. 2, pp. 395-405, 1997.

[9] C. Chuang and N. H. Chan, "Empirical likelihood for autoregressive models, with applications to unstable time series," Statistica Sinica, vol. 12, no. 2, pp. 387-407, 2002.

[10] N. H. Chan, L. Peng, and Y. Qi, "Quantile inference for nearintegrated autoregressive time series with infinite variance," Statistica Sinica, vol. 16, no. 1, pp. 15-28, 2006.

[11] J. Li, W. Liang, S. He, and X. Wu, "Empirical likelihood for the smoothed LAD estimator in infinite variance autoregressive models," Statistics \& Probability Letters, vol. 80, no. 17-18, pp. 1420-1430, 2010.

[12] J. Li, W. Liang, and S. He, "Empirical likelihood for LAD estimators in infinite variance ARMA models," Statistics \& Probability Letters, vol. 81, no. 2, pp. 212-219, 2011.

[13] P. J. Brockwell and R. A. Davis, Time series: Theory and Methods, Springer-Verlag, New York, NY, USA, 2nd edition, 1991.

[14] P. C. B. Phillips, "A shortcut to LAD estimator asymptotics," Econometric Theory, vol. 7, no. 4, pp. 450-463, 1991.

[15] B. W. Silverman, Density Estimation for Statistics and Data Analysis, Chapman \&Hall, London, UK, 1986.

[16] V. H. de la Peña, "A general class of exponential inequalities for martingales and ratios," The Annals of Probability, vol. 27, no. 1, pp. 537-564, 1999.

[17] J. Qin and J. Lawless, "Empirical likelihood and general estimating equations," The Annals of Statistics, vol. 22, no. 1, pp. 300325,1994 


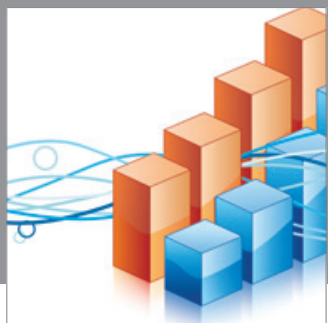

Advances in

Operations Research

mansans

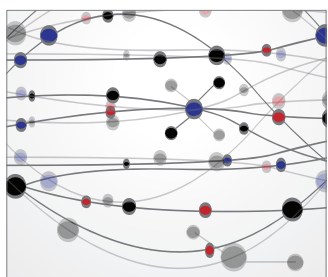

The Scientific World Journal
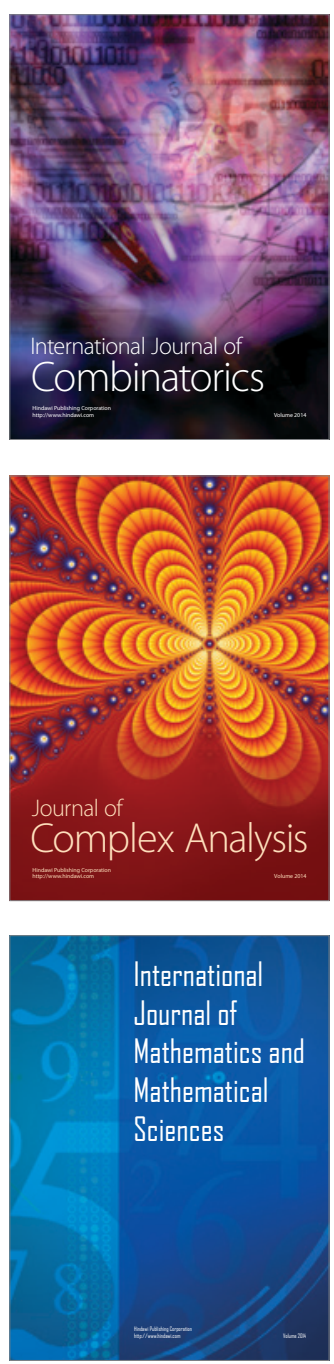
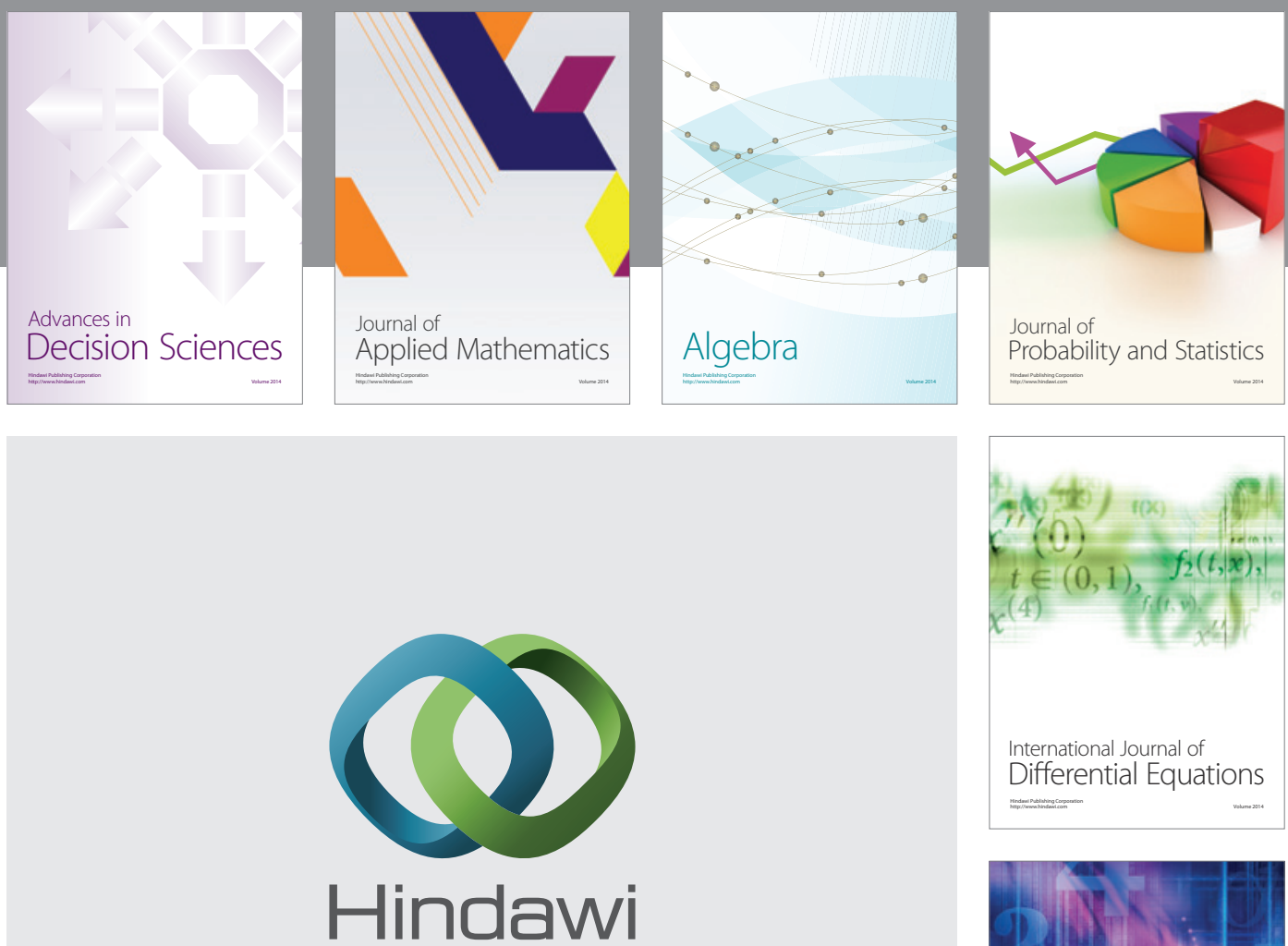

Submit your manuscripts at http://www.hindawi.com
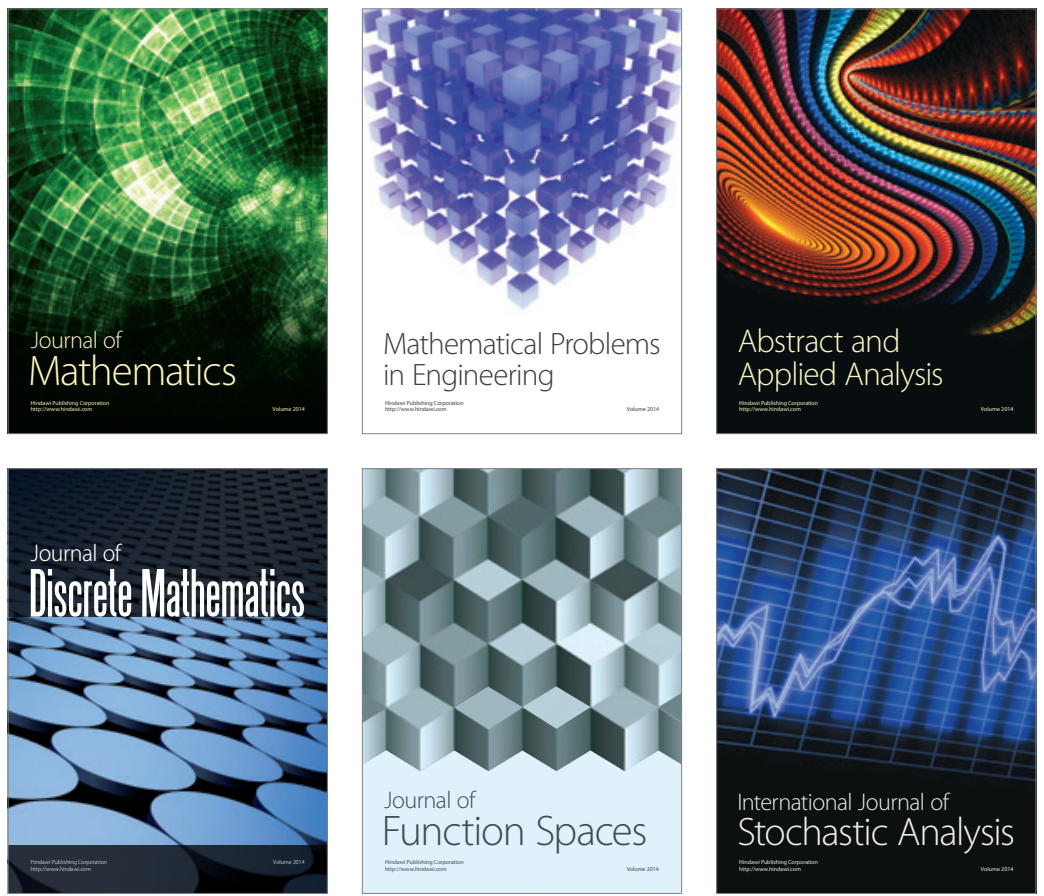

Journal of

Function Spaces

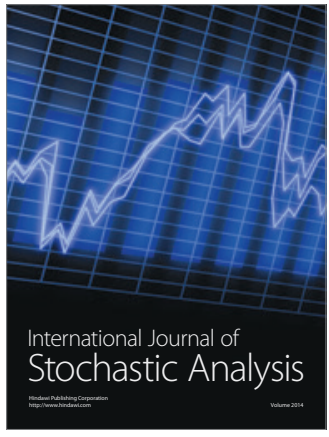

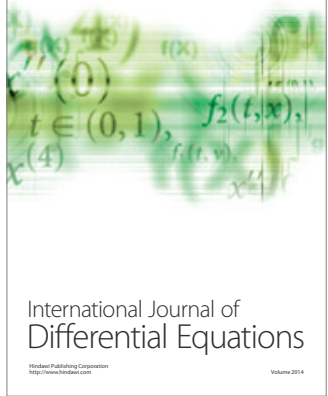
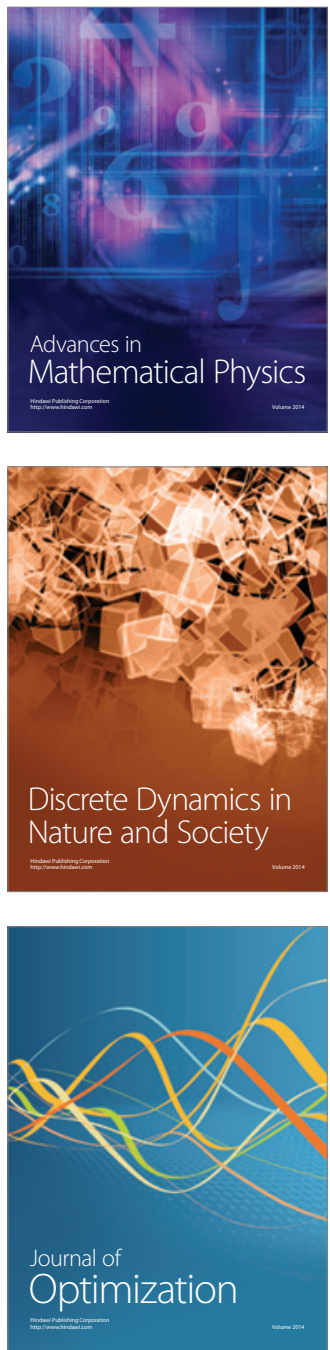